\title{
Population structure and antibiotic resistance of Acinetobacter DNA group 2 and 13TU isolates from hospitals in the UK
}

\author{
RICHARD P. SPENCE, KEVIN J. TOWNER, CAROLINE J. HENWOOD*, DOROTHY JAMES*, \\ NEIL WOODFORD* and DAVID M. LIVERMORE* \\ Molecular Diagnostics and Typing Unit, Public Health Laboratory, Queens Medical Centre, Nottingham \\ NG7 $2 \mathrm{UH}$ and ${ }^{*}$ Antibiotic Resistance Monitoring and Reference Laboratory, Central Public Health Laboratory, \\ London NW9 5 HT
}

\begin{abstract}
A total of 287 Acinetobacter isolates belonging to DNA groups 2 (A. baumannii) and 13TU was collected consecutively from 46 hospitals and typed by randomly amplified polymorphic DNA fingerprinting with primers DAF-4 and ERIC-2. With a similarity coefficient of $\geqslant 72 \%$ as a cut-off value, 37 clusters of genotypically similar isolates (genotypes) were recognised. Four major clusters, found in 15, 12, 12 and 8 hospitals respectively, accounted for $42 \%$ of isolates, but only three of these predominant clusters were associated with outbreaks of infection in individual hospitals. Many of the isolates were resistant to multiple antibiotics, including expanded-spectrum $\beta$-lactam agents, aminoglycosides, tetracyclines and fluoroquinolones, but $\mathbf{7 9 8} \%$ remained susceptible to carbapenems and colistin. Overall, the study demonstrated that a heterogeneous population of Acinetobacter DNA group 2 and 13TU isolates, frequently showing multiple resistance to antibiotics, was causing infections in UK hospitals, and that four predominant genotypes appeared to have disseminated among geographically distinct locations.
\end{abstract}

\section{Introduction}

During the past 25 years, certain species belonging to the genus Acinetobacter have emerged as important causes of nosocomial infection, particularly ventilatorassociated pneumonia, bacteraemia, secondary meningitis and urinary tract infections [1]. A recent European study found that Acinetobacter spp. are the eighth most common cause of nosocomial pneumonia [2]. DNA group 2 (A. baumannii) accounts for the vast majority of these cases [2] and is the genomic species that has been associated most frequently with outbreaks of colonisation and infection in hospitals [1]. Isolates belonging to the closely related DNA group 13TU (not yet given a species name) have also been implicated in a number of outbreaks in intensive care units (ICUs) [1]. Infections caused by A. baumannii and 13TU strains can be extremely difficult to treat because the

Received 2 April 2002; revised version received 7 July 2002; accepted 23 July 2002.

Corresponding author: Dr K. J. Towner (e-mail ktowner@, trent.phls.nhs.uk). bacteria frequently possess multiple antibiotic resistance mechanisms and have a propensity to spread between patients. Therefore, efficient control of Acinetobacter outbreaks is a major challenge for infection control teams. Future control requires knowledge of the population structure of these organisms so that the dynamics of spread of endemic and epidemic strains can be investigated and appropriate control measures can be introduced.

Against this background, the main aim of the present study was to determine the overall population structure of a large collection of clinically significant Acinetobacter DNA group 2 and group 13TU isolates obtained from 46 hospitals in the UK. Numerous different molecular methods have been used to type isolates of Acinetobacter spp. [1, 3, 4], but the large number of isolates to be examined in this study necessitated the use of a rapid and simple typing technique. Randomly amplified polymorphic DNA (RAPD) fingerprinting was chosen because it has been shown to be particularly useful and reproducible with isolates of Acinetobacter spp. [4-6]. The study also aimed to correlate the population structure with antibiotic 
resistance data to determine whether specific resistances were associated with the ability of particular strains to cause outbreaks.

\section{Materials and methods}

\section{Bacterial strains}

As part of a larger survey of antibiotic resistance in Acinetobacter spp. by the Public Health Laboratory Service [7], 287 isolates belonging to DNA groups 2 and $13 \mathrm{TU}$ were obtained from clinical specimens in 46 hospitals throughout the UK between Nov. 1999 and Jan. 2001. Each hospital collected up to 25 consecutive isolates of Acinetobacter from clinical specimens. Demographic data such as patient age, sex, site of isolation and ward type were collated at the Antibiotic Resistance Monitoring and Reference Laboratory, Central Public Health Laboratory, London. Replicate isolates from individual patients were excluded, but some isolates were stated by the collecting hospitals to be associated with outbreaks of infection, whereas others were from sporadic cases. Initial identification of isolates to the genomic species level was by tDNA fingerprinting, as described previously [8], except that amplification products were separated through agarose $1.5 \% \mathrm{w} / \mathrm{v}$ gels at $120 \mathrm{~V} / 200 \mathrm{~mA}$ and were visualised by staining with ethidium bromide $5 \mathrm{mg} / \mathrm{L}$ for $30 \mathrm{~min}$. Discrimination between genomic species 2 and 13TU was confirmed by amplified fragment length polymorphism (AFLP) fingerprinting [9]. Strain RUH 2037 (kindly supplied by Dr L. Dijkshoorn, Leiden University Medical Centre, The Netherlands) was used as a reproducibility control in RAPD experiments.

\section{Susceptibility testing}

Antibiotic MICs for each isolate were determined as part of a previous study [7]. Interpretation of antibiotic susceptibilities was with the breakpoints recommended for Acinetobacter spp. and Enterobacteriaceae by the British Society for Antimicrobial Chemotherapy (BSAC) Working Party $[10,11]$. An exception was sulbactam, for which, in the absence of a BSAC breakpoint, resistance was defined by an MIC of $\geqslant 16 \mathrm{mg} / \mathrm{L}$ [12].

\section{RAPD fingerprinting}

RAPD fingerprints were generated with primers DAF-4 and ERIC-2, as described previously [13]. Amplification products were separated through agarose $2 \% \mathrm{w} / \mathrm{v}$ gels at $120 \mathrm{~V} / 200 \mathrm{~mA}$, with bromophenol blue as the tracking dye. An external reference standard (100-bp DNA ladder; Amersham Pharmacia Biotech, Little Chalfont, Bucks) was run in every sixth track of each gel. DNA fragments were visualised by staining with ethidium bromide $5 \mathrm{mg} / \mathrm{L}$ for $30 \mathrm{~min}$ once the tracking dye had migrated for $10 \mathrm{~cm}$. Gels were recorded and stored as tagged image files with UVIsave image capture equipment (UVItec, Cambridge, Cambs).

Relationships among isolates were examined by analysing the RAPD fingerprints of each isolate with BioNumerics software (Applied Maths, Kortrijk, Belgium). Tagged image files were first converted and normalised in relation to the external reference standards on the same gel, so as to allow the subsequent merging of data from different gels. The software was then used with the Dice coefficient to calculate levels of similarity between fingerprints. Cluster analysis was performed by the unweighted pair group method with arithmetical averages (UPGMA). Each isolate was examined twice, with DNA extracts prepared on different days. Each batch of RAPD experiments included strain RUH 2037 as a reproducibility standard, as well as a negative control containing all components except template DNA. A cut-off value of $\geqslant 72 \%$ similarity - shown previously in similar RAPD experiments to distinguish between unrelated genotypes of $A$. baumannii $[5,13]$ - was used to differentiate clusters of unrelated isolates. Clustering of molecular size markers (100-bp ladder) was used as a measure of the level of inherent variability following merging and clustering of data from different gels. All typing experiments and clustering of fingerprint data were initially performed 'blind' and were correlated with the epidemiological, clinical and susceptibility data only at the conclusion of the study.

\section{Statistical analyses}

The significance of differences in antibiotic susceptibility between outbreak-related and sporadic isolates was examined with the $\chi^{2}$ test, used with Yates' correction; $\mathrm{p}<0.05$ was taken to indicate significance.

\section{Results}

\section{Source of isolates}

Of the 287 DNA group 2 and 13TU isolates included in the study, $60 \%$ (172) were from males, 37\% (106) from females and 3\% (9) were of unknown origin. The average age (51 years) of male and female patients in the study was identical, with age ranges of $0-98$ years and 0-90 years, respectively. The specimens from which the isolates were obtained were blood $(24.7 \%)$, sputum $(18.1 \%)$, wounds $(15 \%)$, aspirates $(7.7 \%)$, burns $(7.0 \%)$, urine samples (5.2\%) and unknown/ others $(22.3 \%)$. ICUs were the source of $37.6 \%$ of the isolates, compared with burns units $(10.1 \%)$, surgical wards $(9.8 \%)$, general medicine wards $(7.3 \%)$ and unknown/others $(35.2 \%)$.

\section{$R A P D$ fingerprinting and clustering of isolates}

The fingerprints generated with primer DAF-4 comprised up to 11 DNA bands, ranging in size from 150 
to $2000 \mathrm{bp}$. Each isolate tested yielded a reproducible fingerprint pattern with DNA extracts prepared on different days. For DAF-4 RAPD, the molecular size marker standards from different gels clustered at $\geqslant 82.3 \%$ similarity with a band position tolerance of $1 \%$ (i.e., above the proposed cut-off value of $\geqslant 72 \%$ similarity for defining unrelated genotypes). In total, 37 clusters (containing 272 isolates) were distinguished by primer DAF-4 at the cut-off value of $\geqslant 72 \%$ similarity (Fig. 1); 15 isolates were classed as sporadic on the grounds that they did not cluster with any other isolate at $\geqslant 72 \%$ similarity.

Most clusters comprised $<10$ isolates; however, there were four large clusters (designated as DAF-4 clusters $3,10,14,32$ ) that each contained $\geqslant 20$ isolates (Fig. 1). In total, $121(42 \%)$ isolates from 25 hospitals belonged to one of these four major clusters. Two of these major clusters (clusters 10 and 14) contained only Acinetobacter DNA group 2 isolates, whereas the other two (clusters 3 and 32) contained only isolates belonging to Acinetobacter DNA group 13TU. Cluster 3 comprised 23 isolates from 15 hospitals spread throughout England and Wales. Cluster 10 included 38 isolates from 12 hospitals located in the Midlands, West and South-East of England, but most (29 of 38) of the isolates included in this cluster originated from hospitals in London and the South-East. Isolates in cluster 14 were obtained from 11 hospitals in England and Wales, although many (17 of 25) of these isolates were from hospitals in London and the South-East. Isolates in cluster 32 were found in eight hospitals in England, Wales and Scotland, but most (25 of 36) came from two Scottish hospitals. The 166 isolates that did not belong to the four major clusters comprised 33 clusters and 15 sporadic isolates, and were distributed among 42 hospitals in England, Wales and Scotland.

Groups of three or more isolates from a single hospital which displayed the same RAPD fingerprints were considered to be potential outbreak strains. Such organisms were obtained from $13(28.2 \%)$ of the 46 hospitals and comprised $35.5 \%$ (102) of the 287 isolates examined. These potential outbreak isolates belonged to 10 DAF-4 clusters (Table 1) and were reexamined with primer ERIC-2 to see whether the same clustering relationships were observed. As found with DAF-4, each isolate tested yielded a reproducible fingerprint pattern with DNA extracts prepared on different days. After merging of gel data, the molecular size standards from each ERIC-2 gel clustered at a level of $92.3 \%$ similarity with a band position tolerance of $1 \%$ (i.e., above the proposed cut-off value of $\geqslant 72 \%$ similarity for defining unrelated genotypes). When the clusters obtained with ERIC-2 were compared with those obtained with DAF-4, a high degree of correlation was observed. Thus, for example, each of the three major DAF-4 clusters involved in potential outbreaks (clusters 10, 14 and 32) correlated with a distinct ERIC-2 cluster, indicating that the relationships ob-
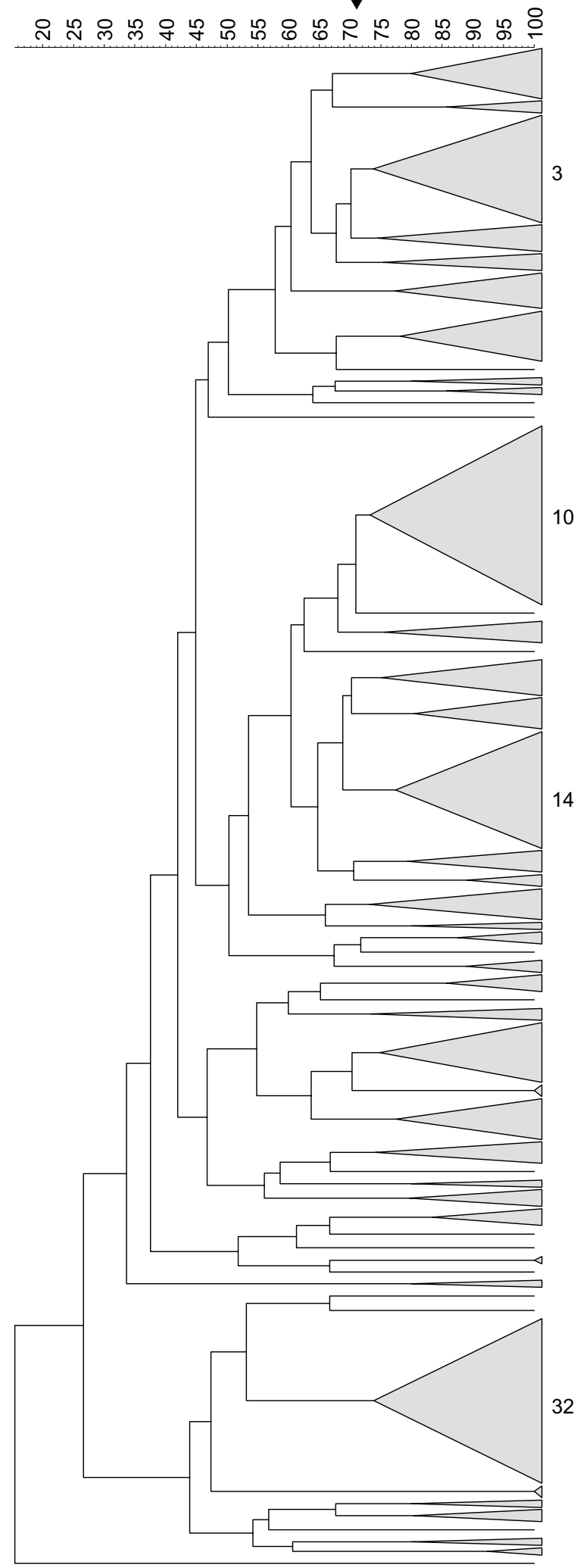

Fig. 1. Dendrogram illustrating the DAF-4 RAPD clustering relationships observed among 287 isolates belonging to Acinetobacter DNA groups 2 and $13 \mathrm{TU}$ obtained from UK hospitals. Cluster relationships were calculated with BioNumerics software by the Dice coefficient and UPGMA method. The arrowhead indicates the position of the previously established $<72 \%$ similarity cut-off point used to differentiate clusters [5, 12]. Clusters labelled $3,10,14$ and 32 are the four predominant clusters identified with primer DAF-4. Clusters 10 and 14 contained only DNA group 2 isolates, while clusters 3 and 32 contained only DNA group 13TU isolates. 
Table 1. Distribution of outbreak-associated Acinetobacter DNA group 2 and $13 \mathrm{TU}$ isolates

\begin{tabular}{|c|c|c|c|c|c|c|c|c|c|c|}
\hline \multirow{2}{*}{$\begin{array}{l}\text { DAF-4 } \\
\text { cluster }\end{array}$} & \multirow{2}{*}{$\begin{array}{c}\text { Genomic } \\
\text { species }\end{array}$} & \multirow{2}{*}{$\begin{array}{c}\text { Total } \\
\text { number } \\
\text { of isolates }\end{array}$} & \multirow{2}{*}{$\begin{array}{l}\text { Isolates } \\
\text { involved } \\
\text { in } \\
\text { outbreaks }\end{array}$} & \multicolumn{7}{|c|}{ Number of hospitals with outbreaks } \\
\hline & & & & SE & $\mathrm{E}$ & Mid & SW & $\mathrm{N}$ & Sco & Wal \\
\hline 1 & 2 & 11 & 6 & 0 & 0 & 2 & 0 & 0 & 0 & 0 \\
\hline 6 & 2 & 8 & 4 & 0 & 0 & 0 & 0 & 0 & 0 & 1 \\
\hline 7 & 2 & 10 & 3 & 1 & 0 & 0 & 0 & 0 & 0 & 0 \\
\hline 10 & 2 & 38 & 30 & 5 & 0 & 1 & 0 & 0 & 0 & 0 \\
\hline 13 & 2 & 7 & 3 & 0 & 0 & 0 & 0 & 1 & 0 & 0 \\
\hline 14 & 2 & 25 & 13 & 2 & 0 & 0 & 0 & 0 & 0 & 0 \\
\hline 21 & 2 & 4 & 4 & 0 & 1 & 0 & 0 & 0 & 0 & 0 \\
\hline 23 & 2 & 13 & 6 & 2 & 0 & 0 & 0 & 0 & 0 & 0 \\
\hline 25 & 2 & 9 & 5 & 1 & 0 & 0 & 0 & 0 & 0 & 0 \\
\hline 32 & $13 \mathrm{TU}$ & 36 & 28 & 0 & 0 & 0 & 0 & 0 & 2 & 1 \\
\hline
\end{tabular}

SE, London and South-East England; E, East of England; Mid, Midlands; SW, South-West England; N, North of England; Sco, Scotland; Wal, Wales.

served between the potential outbreak isolates were not artefacts generated by the choice of a particular RAPD primer or by the RAPD technique itself. Of the potential outbreak isolates, $69.6 \%$ (71 of 102$)$ belonged to these three clusters (Table 1). Of the 38 isolates belonging to DAF-4 cluster 10, $30 \quad(78.9 \%)$ were associated with outbreaks of infection in six hospitals. In the case of DAF-4 cluster $14,13(52 \%)$ of the 25 isolates were associated with outbreaks of infection in two hospitals. Of 36 isolates in DAF-4 cluster 32, 28 $(77.8 \%)$ came from two Scottish hospitals and one from Wales. The remaining 31 outbreak-associated isolates came from eight hospitals and belonged to seven different clusters (Table 1).

Of note was the fact that none of the potential outbreaks involved isolates belonging to the major DAF-4 cluster 3 . Thus, although isolates in this cluster were isolated from 15 hospitals throughout England and Wales, there was no evidence for this lineage causing outbreaks of infection within individual hospitals. However, when DAF-4 cluster 3 was re-examined with primer ERIC-2, three ERIC-2 sub-clusters were found, indicating that the isolates in DAF-4 cluster 3 may be more diverse than suggested by RAPD fingerprinting with DAF-4 alone.

\section{Antimicrobial susceptibilities}

More than $80 \%$ of the isolates included in the study were resistant to cefotaxime, ceftazidime and tetracycline, but most isolates remained susceptible to carbapenems and colistin, which are the agents currently used to treat most multi-drug-resistant Acinetobacter infections (Table 2). Outbreak-related isolates showed higher rates of resistance to all antibiotics tested, with the exceptions of colistin and sulbactam; however, this difference was not significant for any antibiotic considered ( $p \geqslant 0.05$ in each case).

Some minor variations in resistance profiles were observed among isolates belonging to the same cluster. Within the four main DAF-4 clusters, isolates were generally resistant to cephalosporins, penicillins and

Table 2. Prevalence of antibiotic resistance among Acinetobacter DNA group 2 and 13TU isolates from UK hospitals

\begin{tabular}{lccc}
\hline & \multicolumn{3}{c}{ Percentage of isolates resistant* } \\
\cline { 2 - 4 } Antibiotic & $\begin{array}{c}\text { Total } \\
(\mathrm{n}=287)\end{array}$ & $\begin{array}{c}\text { Outbreak-related } \\
(\mathrm{n}=102)\end{array}$ & $\begin{array}{c}\text { Non-outbreak related } \\
(\mathrm{n}=185)\end{array}$ \\
\hline Amikacin & 23.1 & 27.5 & 20.5 \\
Ciprofloxacin & 48.4 & 60.8 & 41.6 \\
Colistin & 1.1 & 0 & 1.6 \\
Cefotaxime & 95.0 & 100 & 92.4 \\
Ceftazidime & 87.2 & 96.0 & 82.1 \\
Gentamicin & 45.6 & 55.9 & 40.0 \\
Imipenem & 1.8 & 2.9 & 1.1 \\
Meropenem & 0.7 & 0.9 & 0.5 \\
Piperacillin & 69.8 & 86.3 & 60.5 \\
Piperacillin/tazobactam & 40.4 & 49.0 & 35.7 \\
Sulbactam & 11.7 & 9.8 & 13.0 \\
Tetracycline & 86.5 & 89.2 & 84.9 \\
\hline
\end{tabular}

* Susceptibilities were interpreted with the breakpoints recommended for Acinetobacter spp. by the British Society for Antimicrobial Chemotherapy [10,11]. Differences in the prevalence of resistance between outbreak-related and non-outbreak isolates were not significant $(\mathrm{p}>0.05)$. 
tetracycline, with isolates belonging to clusters 10 and 14 additionally showing resistance to aminoglycosides and quinolones. Isolates not belonging to the four major clusters varied from fully sensitive to highly resistant, but the isolate numbers in the individual clusters were too small to allow firm conclusions to be reached. Three outbreak-associated isolates in cluster 10 (all from a single hospital) and two non-outbreak isolates in cluster 14 were resistant to imipenem. Two isolates from different hospitals (one outbreak-related isolate belonging to cluster 10 that was also imipenemresistant, and one non-outbreak isolate in cluster 13) were resistant to meropenem.

\section{Discussion}

As reported previously [4-6], RAPD fingerprinting was found to be a reliable and reproducible typing technique for Acinetobacter DNA group 2 and 13TU isolates. It is important to note that the highly standardised methodology [13] employed in this study is essential to achieve the necessary level of reproducibility with the RAPD technique. However, providing that the technique is carefully standardised, it has been established previously [4] that RAPD typing of Acinetobacter spp. provides robust results that are comparable with those obtained by other typing techniques such as pulsed-field gel electrophoresis. In comparison, conventional phenotypic tests are generally inadequate even to distinguish between different Acinetobacter DNA groups [1], while biotyping gives poor discrimination between individual strains of DNA groups 2 and 13TU [4].

The RAPD typing results obtained in the study indicated that there was a heterogeneous population of Acinetobacter DNA group 2 and 13TU isolates in the UK hospitals studied. However, four main clusters of isolates were found within this population, and members of three of these clusters were implicated in outbreaks of infection in particular locations, in addition to being isolated sporadically at other locations. For example, of the 22 isolates examined from a single hospital in Scotland, 18 belonged to DAF-4 cluster 32 when analysed by RAPD fingerprinting. Isolates in this cluster, all of which belonged to Acinetobacter DNA group 13TU, were also found at a second hospital in Scotland and one in Wales. A previous study similarly identified a single strain of Acinetobacter DNA group $13 \mathrm{TU}$ as the main cause of Acinetobacter infections in Edinburgh Royal Infirmary over a 3-year period in the 1990s [14]. In contrast, other hospitals appeared to have diverse populations of Acinetobacter DNA group 2 and 13TU isolates. For example, of 15 isolates examined from a hospital in the Midlands, 13 belonged to different clusters, suggesting that there were a number of different strains circulating in this particular hospital that were capable of causing infections. Similar contrasting epidemiological relation- ships among Acinetobacter isolates from different geographical locations have been reported previously [15], possibly reflecting patient mix or the efficiency of different local control of infection policies and procedures.

Three of the four major clusters identified in this study seemed to have spread in specific regions of the country. For example, isolates in DAF-4 cluster 32 were predominantly collected from Scottish hospitals, whereas isolates in DAF-4 clusters 10 and 14 were found mainly in hospitals in London and the SouthEast of England. DAF-4 cluster 3 appeared to be different from the other predominant clusters, in that its constituent isolates were not concentrated in any specific region of the country. Furthermore, the isolates in this cluster were not associated with outbreaks of infection in individual hospitals, initially suggesting that not all widely disseminated strains of Acinetobacter DNA group 2 or $13 \mathrm{TU}$ are equally capable of causing such outbreaks. However, further RAPD typing experiments with primer ERIC-2 revealed evidence for a limited amount of diversity within the cluster, indicating that this cluster was not as homogeneous as suggested by RAPD typing with a single primer.

The evidence of inter-hospital spread of Acinetobacter DNA group 2 and 13TU strains supports the conclusions of several other European studies that have examined the epidemiology of $A$. baumannii $[3,16]$. However, the potentially 'epidemic' strains circulating in UK hospitals do not seem to be the same as the two major epidemic strains found in other European countries (personal unpublished data). Furthermore, a recent RAPD fingerprinting study of $A$. baumannii isolates from 49 medical centres across the USA found no evidence of inter-hospital spread [6]. Additional studies are required to determine whether these differences are strain-related or a consequence of specific infection control measures and antibiotic policies implemented in different locations.

As in previous studies of Acinetobacter spp. [6, 7, 1719], many of the nosocomial Acinetobacter DNA group 2 and 13TU isolates in the present study were found to be multiresistant. Strains that predominate in certain hospitals or regions may possess characteristic features that enable them to out-compete other strains. Multiple antibiotic resistance is one such factor that might be expected to give a selective advantage in spread of particular strains $[3,20]$. However, two of the main clusters (DAF-4 clusters 3 and 32) identified in the present study were not highly antibiotic-resistant but, notably, belonged to Acinetobacter DNA group 13TU rather than $A$. baumannii. The significance of this observation is hard to assess at present, because most previous studies have not distinguished between DNA groups 2 and 13TU, and little is known about the general epidemiology of $13 \mathrm{TU}$ isolates. The two other main clusters, comprising isolates of $A$. baumannii, 
were generally multiresistant. Resistance to carbapenems was confined to six isolates belonging to three clusters (10, 13 and 14) from two hospitals in the South-East of England and one in the North-West. Three of these isolates (all belonging to cluster 10) were outbreak-associated, but carbapenem resistance was not found in the remaining 35 isolates belonging to the same cluster, suggesting that resistance was appearing in individual patients who had already acquired the outbreak strain.

It has been proposed that the ability of particular strains to cause outbreaks within and between hospitals is likely to be multifactorial and related to factors other than the simple possession of antibiotic resistance mechanisms. Thus, Koeleman et al. [20] reported that multiple antibiotic resistance and the ability to bind to salivary mucins both correlated with the epidemic behaviour of $A$. baumannii isolates. It is also probable that the ability of particular strains to survive for long periods in the hospital environment enhances their chances of transmission [21,22]. Nevertheless, certain antibiotic resistance determinants, once acquired, may favour repeated selection of specific strains in the hospital setting. Other possible factors involved in determining the outbreak potential of strains remain to be elucidated.

We are indebted to colleagues at all the clinical microbiology laboratories that collected the strains included in the study, to $\mathrm{L}$. Dijkshoorn for the gift of strain RUH 2037, and to T. van der Reijden for teaching R.P.S. the AFLP fingerprinting technique.

\section{References}

1. Bergogne-Bérézin E, Towner KJ. Acinetobacter spp. as nosocomial pathogens: microbiological, clinical, and epidemiological features. Clin Microbiol Rev 1996; 9: 148-165.

2. Fluit AC, Schmitz FJ, Verhoef $J$ and the European SENTRY Participant Group. Frequency of isolation of pathogens from bloodstream, nosocomial pneumonia, skin and soft tissue, and urinary tract infections occurring in European patients. Eur $J$ Clin Microbiol Infect Dis 2001; 20: 188-191.

3. Dijkshoorn L, Aucken H, Gerner-Smidt P et al. Comparison of outbreak and nonoutbreak Acinetobacter baumannii strains by genotypic and phenotypic methods. J Clin Microbiol 1996; 34: 1519-1525.

4. Webster CA, Towner KJ, Humphreys H, Ehrenstein B, Nartung $\mathrm{D}$, Grundmann H. Comparison of rapid automated laser fluorescence analysis of DNA fingerprints with four other computer-assisted approaches for studying relationships between Acinetobacter baumannii isolates. $J$ Med Microbiol 1996; 44: 185-194.

5. Grundmann H, Schneider C, Tichy HV et al. Automated laser fluorescence analysis of randomly amplified polymorphic DNA: a rapid method for investigating nosocomial transmission of Acinetobacter baumannii. J Med Microbiol 1995; 43: 446-451.
6. Wisplinghoff $\mathrm{H}$, Edmond MB, Pfaller MA, Jones RN, Wenzel RP, Seifert H. Nosocomial bloodstream infections caused by Acinetobacter species in United States hospitals: clinical features, molecular epidemiology, and antimicrobial susceptibility. Clin Infect Dis 2000; 31: 690-697.

7. Henwood CJ, Gatwood T, Warner M et al. Antibiotic resistance among clinical isolates of Acinetobacter in the UK, and invitro evaluation of tigecycline (GAR-936). $J$ Antimicrob Chemother 2002; 49: 479-487.

8. Ehrenstein B, Bernards AT, Dijkshoorn L et al. Acinetobacter species identification by using tRNA spacer fingerprinting. $J$ Clin Microbiol 1996; 34: 2414-2420.

9. Janssen P, Maquelin K, Coopman R et al. Discrimination of Acinetobacter genomic species by AFLP fingerprinting. Int $J$ Syst Bacteriol 1997; 47: 1179-1187.

10. Andrews JM, for the BSAC Working Party on Susceptibility Testing. BSAC standardized disc susceptibility testing method. J Antimicrob Chemother 2001; 48 Suppl S1: 43-57.

11. MacGowan AP, Wise R. Establishing MIC breakpoints and the interpretation of in vitro susceptibility tests. J Antimicrob Chemother 2001; 48 Suppl S1: 17-28.

12. Bello H, Domínguez M, González G et al. In vitro activities of ampicillin, sulbactam and a combination of ampicillin and sulbactam against isolates of Acinetobacter calcoaceticusAcinetobacter baumannii complex isolated in Chile between 1990 and 1998. J Antimicrob Chemother 2000; 45: 712-713.

13. Grundmann HJ, Towner KJ, Dijkshoorn L et al. Multicenter study using standardized protocols and reagents for evaluation of reproducibility of PCR-based fingerprinting of Acinetobacter spp. J Clin Microbiol 1997; 35: 3071-3077.

14. McDonald A, Amyes SGB, Paton R. The persistence and clonal spread of a single strain of Acinetobacter 13TU in a large Scottish teaching hospital. J Chemother 1999; 11: $338-344$.

15. Webster CA, Towner KJ, Saunders GL, Crewe-Brown HH, Humphreys H. Molecular and antibiogram relationships of Acinetobacter isolates from two contrasting hospitals in the United Kingdom and South Africa. Eur J Clin Microbiol Infect Dis 1999; 18: 595-598.

16. Nemec A, Janda L, Melter O, Dijkshoorn L. Genotypic and phenotypic similarity of multiresistant Acinetobacter baumannii isolates in the Czech Republic. J Med Microbiol 1999; 48: 287-296.

17. Corbella X, Montero A, Pujol M et al. Emergence and rapid spread of carbapenem resistance during a large and sustained hospital outbreak of multiresistant Acinetobacter baumannii. $J$ Clin Microbiol 2000; 38: 4086-4095.

18. Gales AC, Jones RN, Forward KR, Liñares J, Sader HS, Verhoef J. Emerging importance of multidrug-resistant Acinetobacter species and Stenotrophomonas maltophilia as pathogens in seriously ill patients: geographic patterns, epidemiological features and trends in the SENTRY Antimicrobial Surveillance Program (1997-1999). Clin Infect Dis 2001; 32 Suppl 2: 104-113.

19. Manikal VM, Landman D, Saurina G, Oydna E, Lal H, Quale J. Endemic carbapenem-resistant Acinetobacter species in Brooklyn, New York: citywide prevalence, interinstitutional spread, and relation to antibiotic usage. Clin Infect Dis 2000; 31: $101-106$.

20. Koeleman JGM, van der Bijl MW, Stoof J, Vandenbroucke CMJE, Savelkoul PHM. Antibiotic resistance is a major risk factor for epidemic behaviour of Acinetobacter baumannii. Infect Control Hosp Epidemiol 2001; 22: 284-288.

21. Jawad A, Seifert H, Snelling AM, Heritage J, Hawkey PM. Survival of Acinetobacter baumannii on dry surfaces: comparison of outbreak and sporadic isolates. J Clin Microbiol 1998; 36: 1938-1941.

22. Wendt C, Dietz B, Dietz E, Ruden H. Survival of Acinetobacter baumannii on dry surfaces. J Clin Microbiol 1997; 35: 1394-1397. 